\title{
Acceptance of noninvasive computed tomography coronary angiography: for a patient-friendly medicine
}

\author{
Ludovico La Grutta - Sabina La Grutta - Massimo Galia • Giuseppe Lo Piccolo • \\ Giovanni Gentile • Giuseppe La Tona • Maria Stella Epifanio • Erica Maffei • \\ Filippo Cademartiri · Rosa Lo Baido $\cdot$ Roberto Lagalla $\cdot$ Massimo Midiri
}

Received: 22 April 2012/ Accepted: 12 July 2012/Published online: 26 November 2013

(C) Italian Society of Medical Radiology 2013

\begin{abstract}
Purpose This study was done to evaluate the psychological state and anxiety of patients undergoing computed tomography coronary angiography (CTCA), and assess their acceptance and satisfaction compared to invasive conventional coronary angiography (CCA).

Materials and methods A total of 442 consecutive patients (282 male; mean age $57.7 \pm 9.5$ years) who underwent CTCA for suspected or known coronary artery disease were evaluated with the Endler Multimodality Anxiety Scales (EMAS) before and after the scan, and a questionnaire administered after the scan. Among the 442 patients, 181 had a history of CCA. Two radiologists assessed the image quality of CTCA.
\end{abstract}

L. La Grutta $(\bowtie) \cdot$ M. Galia $\cdot$ G. Gentile

G. La Tona $\cdot$ R. Lagalla $\cdot$ M. Midiri

Department of Radiology, DIBIMEF, University Hospital

"P. Giaccone", University of Palermo, Via del Vespro 127,

90127 Palermo, Italy

e-mail: ludovicolagrutta@hotmail.it

S. La Grutta · G. Lo Piccolo · M. S. Epifanio

Department of Psychology, University of Palermo, Viale delle

Scienze-Edificio 15, 90100 Palermo, Italy

E. Maffei · F. Cademartiri

Cardio-Vascular Imaging Unit-Giovanni XXIII Hospital, Via

Giovanni XXIII, 7, 31050 Monastier di Treviso (TV), Italy

F. Cademartiri

Department of Radiology and Cardiology, Erasmus Medical

Center, Dr. Molenwaterplein 40, 3015GD Rotterdam,

The Netherlands

R. Lo Baido

Department of Clinical Neuroscience, BIONEC, University of Palermo, Via del Vespro 127, 90127 Palermo, Italy
Results Anxiety was more intense prior to the scan (EMAS score 51.7 vs. 46.7, $p<0.01$ ) and in patients with a history of CCA (EMAS score 55.5 vs. $49.1, p<0.01$ ). Women presented more intense anxiety (EMAS score 59.5 vs. $47.3, p<0.01)$, higher mean heart rate $(63.5 \pm 7.6$ vs. $60.7 \pm 7.3$ beats per minute, $p<0.01)$ and a lower image quality than men $(p<0.0001)$. CTCA proved to be more acceptable than CCA because of accurate preparation, lower concern prior to the examination, negligible pain, higher comfort, and greater overall satisfaction $(p<0.0001)$.

Conclusions Computed tomography coronary angiography is a patient-friendly imaging method because of the minimal perceived discomfort. Anxiety may affect CTCA image quality in women.

Keywords Coronary artery disease - Anxiety . Conventional coronary angiography $\cdot \mathrm{CT}$ coronary angiography

\section{Introduction}

Coronary angiography with computed tomography (CTCA) has emerged as one of the most technologically fascinating imaging tools over the last years [1-3]. CTCA has achieved high sensitivity and negative predictive value in the evaluation of coronary artery disease with applications extending to various clinical settings [4-9] and recently reinforced by the excellent prognostic outcome [10-12].

However, the point of view of the patient and the social acceptance of a method should also be tested for a comprehensive assessment of a modern medical technology [13]. In fact, the success of a method is undeniably 
associated with the intrinsic acceptance of the method by the patients, who are increasingly pursuing noninvasive and outpatient diagnostic examinations [14]. On the one hand, recent studies have shown that noninvasive tools such as CT virtual colonoscopy [15], magnetic resonance (MR) cholangiopancreatography [16], and MR angiography of the carotid arteries [17] are widely accepted by patients more than the traditional invasive approach, on the other hand it is known that conventional coronary angiography (CCA) may cause intense concern and anxiety in the patients and affect their quality of life especially in the hours before the examination, with the need for pharmacological and/or psychological treatment [18-23].

The aim of our study was to define the level of anxiety, using a scientific method, in patients undergoing CTCA and outline the patients' acceptance of the technique.

\section{Materials and methods}

\section{Population}

A total of 442 patients (282 men; mean age, $57.7 \pm 9.5$ years) who underwent 64-slice CTCA between 01 September 2008 and 01 March 2010 at our Department were included in the study. Exclusion criteria were: refusal to provide informed consent, severe renal impairment (creatininaemia $>120 \mathrm{mmol} / \mathrm{l}$ ), known allergy to iodinated contrast media, possible pregnancy, presence of hyperkinetic arrhythmias, severe impairment of respiratory function. Patients underwent CTCA with the following clinical indications: suspected coronary artery disease $(n=299)$, atypical chest pain $(n=152,34 \%)$, typical angina with inconclusive stress test $(n=47,11 \%)$, high cardiovascular risk profile $(n=52,12 \%)$, or candidates for aortic valve replacement surgery $(n=48,11 \%)$; follow-up of myocardial revascularisation procedures performed at least 2 years before CTCA $(n=143)$, including the assessment of the patency of proximal stents $(n=75$, $17 \%)$ and bypass grafts $(n=68,15 \%)$. A history of CCA was present in 181 patients.

All patients underwent the Endler Multidimensional Assessment of Anxiety (Endler Multimodality Anxiety Scales, EMAS) before and after CTCA, and a multiple choice questionnaire with a five-point Likert scale for the evaluation of acceptance after CTCA. The ethics committee approved the study protocol and all patients provided written informed consent.

Scan and image reconstruction parameters

All examinations were performed with a 64-slice CT system (Brilliance 64, Philips Medical Systems, Cleveland,
OH, USA). CT scans were performed with the following parameters: slices/collimation $64 / 0.6 \mathrm{~mm}$, rotation time $420 \mathrm{~ms}$, effective temporal resolution (with $180^{\circ}$ algorithm) $210 \mathrm{~ms}, 120 \mathrm{kV}, 800-1040 \mathrm{mAs}$, table feed $11.9 \mathrm{~mm} / \mathrm{s}$, effective slice thickness $0.8 \mathrm{~mm}$, reconstruction increment $0.4 \mathrm{~mm}$, field of view (FOV) $140-240 \mathrm{~mm}$ (extended cranially only for evaluating ascending aorta aneurysms and by-pass grafts). Patients with a heart rate $>65$ beats per minute (bpm) were given a dose of 20-40 mg of propranolol by mouth (Inderal, AstraZeneca Reims, Reims Cedex, France) $1 \mathrm{~h}$ prior to the scan to lower the heart rate. In the leaflet-informing patients on how to prepare for the examination, treatment with $20-40 \mathrm{mg}$ propranolol twice daily under physician supervision was recommended in the 3 days prior to the examination to lower and stabilise the heart rate in patients with tachycardia. All patients received a $2.5-\mathrm{mg}$ dose of diazepam (Tranquirit, Aventis Pharma, Waterford, Ireland) $1 \mathrm{~h}$ prior to the scan. A bolus of $100-120 \mathrm{ml}$ of nonionic iodinated contrast agent (iomeprol, Iomeron 400, Bracco, Milan, Italy), according to the scan range, was administered at an injection rate of $5 \mathrm{ml} / \mathrm{s}$ using an automatic injector (Stellant, MedRAD, Pittsburgh, PA, USA) connected to an 18 -gauge needle cannula placed in a right antecubital vein. Intracoronary enhancement was optimised by using the bolus-tracking technique, with a region of interest (ROI) positioned at the level of the ascending aorta in order to synchronise the start of the scan with the arrival of the contrast agent. The scan started automatically with an $8 \mathrm{~s}$ delay after a threshold of $120 \mathrm{HU}$ was reached within the ROI. Data were retrospectively reconstructed in the enddiastolic phase $(65-80 \%$ of the RR interval) and endsystolic phase (40-45\%).

Two radiologists with experience in CTCA assessed in consensus image quality with a four-point qualitative scale distinguishing examinations with excellent, good, sufficient, and insufficient image quality [24].

Assessment of anxiety and questionnaire of acceptance

The Endler test for the assessment of multidimensional anxiety (EMAS, Endler Multidimensional Anxiety Scale, 1991, Italian translation 1996, O.S. Organizzazioni Speciali, Florence, Italy) was administered to all patients, before the CTCA and in any case immediately after the administration of diazepam; $2 \mathrm{~h}$ after the scan, the Endler test was administered again, in conjunction with a questionnaire rating the acceptance of the diagnostic examination. The tests were administered by a clinical psychologist so as to address potential problems arising during completion of the test and questionnaire.

The EMAS test is a "pencil and paper" questionnaire of simple administration which measures the level of state 
anxiety, trait anxiety, and perception of anxiety during a threat inherent in the present situation [25]. The EMAS test, while maintaining the traditional constructs of the distinction between state and trait anxiety, represents a multidimensional scale of anxiety that can accurately assess individual responses and anxiety reactions in different situations. In addition, the EMAS test was developed and standardised on samples representative of the Italian population. The EMAS test is composed of three parts: the EMAS-State (EMAS-S), which evaluates state anxiety in relation to autonomicemotional and cognitive components; the EMAS-trait (EMAS-T), which assesses anxiety proneness in four different general contexts [social evaluation (SE), physical danger (PD), ambiguous situations (AM), and daily routine (DR)]; the EMAS-perception of the situation (EMAS-P), fundamental control in a research context, which represents a measure of the respondent's subjective perception of the type of situation and degree of threat evoked by that situation at the time of testing [25]. All raw EMAS scores were converted to standard $T$ points or percentiles.

At the end of CTCA, a questionnaire to assess acceptance was proposed to all patients. In particular, the following aspects were evaluated: preparation and information before the imaging examination, degree of preceding concern, comfort, helplessness during the examination, pain experienced, degree of overall satisfaction. Evaluation was performed with a five-point qualitative Likert scale: very low, low, moderate, high, very high [26]. The same questionnaire was formulated also with reference to $\mathrm{CCA}$ and given to the 181 patients who reported having a history of CCA imaging.

\section{Statistical analysis}

The results of the EMAS-S test in all patients before (EMAS-SI) and after the performance of CTCA (EMASSII) were compared by using Student's $t$ test $(p<0.01)$, further dividing subjects into subgroups based on gender and presence of CCA history. Confidence intervals were calculated at $99 \%$. Multiple regression analysis was performed between the level of state anxiety and heart rate values immediately before, during, and immediately after CTCA. State anxiety before the scan was controlled with the EMAS-P scale of perception of the situation here and now. The $X^{2}$ test was used to compare image quality of CTCA in men and women, as well as the qualitative variables of the acceptance questionnaire of CTCA and CCA $(p<0.0001)$.

\section{Results}

The state anxiety measured before CTCA was more intense than that measured after CTCA (EMAS-SI score 51.7 vs.
EMAS-SII score 46.7, $p<0.01$, Fig. 1), and correlated with the heart rate $\left(r^{2}=0.4672\right)$. The average heart rates were $61.7 \pm 7.5 \mathrm{bpm}$ immediately before, $62.9 \pm 8.6 \mathrm{bpm}$ during, $75.2 \pm 13.7 \mathrm{bpm}$ immediately after CTCA. Overall image quality was excellent in $74 \%$ of cases $(n=326$ patients), good in $14 \%(n=62)$, sufficient in $10 \%$ $(n=46)$, and poor in $2 \%(n=8)$. Anxiety was higher in women than in men (EMAS-SI score 59.5 vs. 47.3, $p<0.01)$ in all components measured, apart from trait anxiety related to physical danger $(p<0.01)$ and daily routine $(p>0.01)$ (Fig. 2). The values of heart rate collected immediately before (men $60.7 \pm 7.3 \mathrm{bpm}$ vs. women $63.5 \pm 7.6 \mathrm{bpm}$ ), during (men $60.3 \pm 8.1 \mathrm{bpm}$ vs. women $67.4 \pm 7.5 \mathrm{bpm}$ ), and after CTCA (men $73.2 \pm 16.3 \mathrm{bpm}$ vs. Women $78.7 \pm 5.1 \mathrm{bpm})$ were significantly higher in women $\left(p<0.01\right.$, men $r^{2}=0.8978$, women $r^{2}=0.5072$ ) (Fig. 3). Image quality of CTCA was higher in men (excellent $n=238,84 \%$; good $n=15$, $5 \%$; sufficient $n=24,9 \%$; poor $n=5,2 \%$ ) than in women (excellent $n=88,55 \%$; good $n=47,29 \%$; sufficient $n=22,14 \%$; poor $n=3,2 \%)(p<0.0001)$.

State anxiety was higher in patients with a history of previous CCA (EMAS-SI score 55.5 vs. 49.1, $p<0.01$ ), achieving similar values in the evaluation performed after CTCA (EMAS-SII score 46.6 vs. 46.7, $p>0.01$ ) (Fig. 4).

Computed tomography coronary angiography showed an excellent acceptance profile, with higher acceptance than CCA in the 181 with a history of CCA in terms of preparation and information before the examination, degree of preceding concern, comfort, helplessness during the examination, pain experienced, degree of overall satisfaction (Tables 1, 2, $p<0.0001$ ).

\section{Discussion}

The technological innovation of the last decades in the field of diagnostic imaging has led to a parallel development of an evaluation system, which aims at identifying the appropriateness, results, and costs of a method. The process of the Health Technology Assessment (HTA) investigates not only the technical reliability and diagnostic performance of a new method, but also aims to assess its prognostic, economic, and social impact, with the primary goal of improving health and wellbeing of the population, by integrating both medical and nonmedical multidisciplinary knowledge and strategies [13]. Therefore, HTA needs to combine clinical and scientific evidence with social, cultural and ethical aspects, largely involving the patients, who are the protagonists and beneficiaries of a health service [27]. It is now recognised that full acceptance of a medical procedure or a diagnostic tool by the patient provides more satisfaction, better clinical results, and 


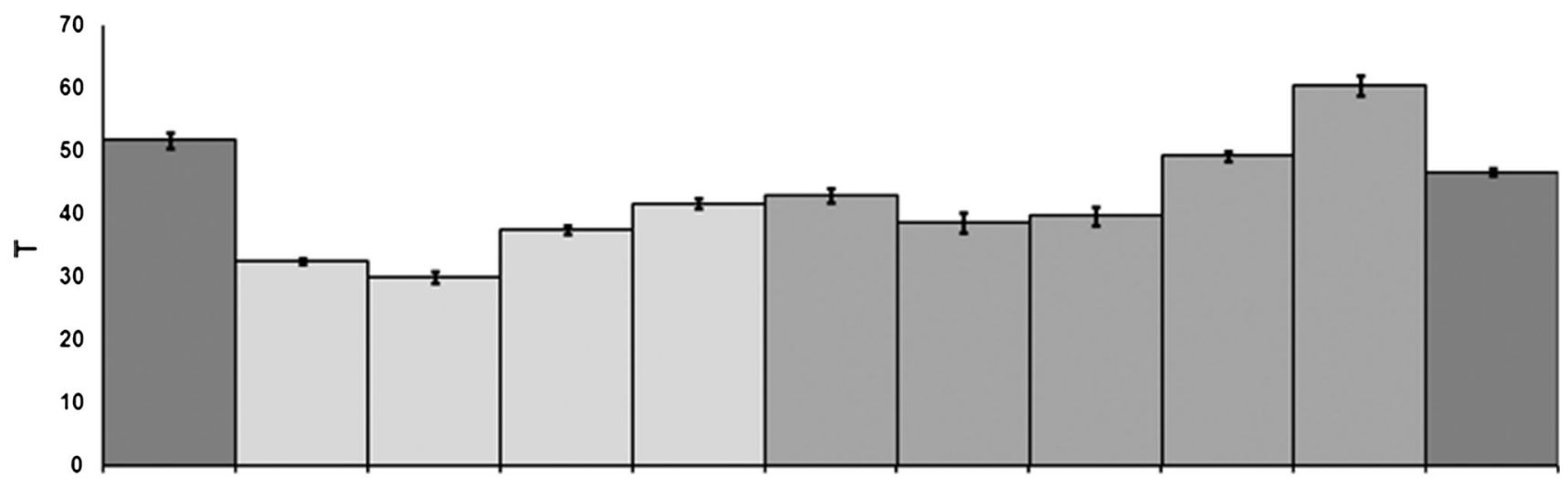

EMAS-S I EMAS.T.SE EMAS.T-PDEMAS-T-AMEMAS-T.DR EMAS.P.1 EMAS.P.2 EMAS.P.3 EMAS.P-4 EMAS.P.5 EMAS-S II

Fig. 1 Assessment with Endler Multimodality Anxiety Scales (EMAS); bars represent confidence intervals at $99 \%$. EMAS-S I, state anxiety before computed tomography coronary angiography (CTCA); EMAS-T-SE, trait anxiety of social evaluation; EMAS-T$\mathrm{PD}$, trait anxiety of physical danger; EMAS-T-AM, trait anxiety of ambiguous situation; EMAS-T-DR, trait anxiety of daily routine;
EMAS-P-1, perception of anxiety during SE; EMAS-P-2, perception of anxiety during PD; EMAS-P-3, perception of anxiety during AM; EMAS-P-4, perception of anxiety during DR; EMAS-P-5, perception of anxiety inherent in the present situation; EMAS-S II, state anxiety after CTCA

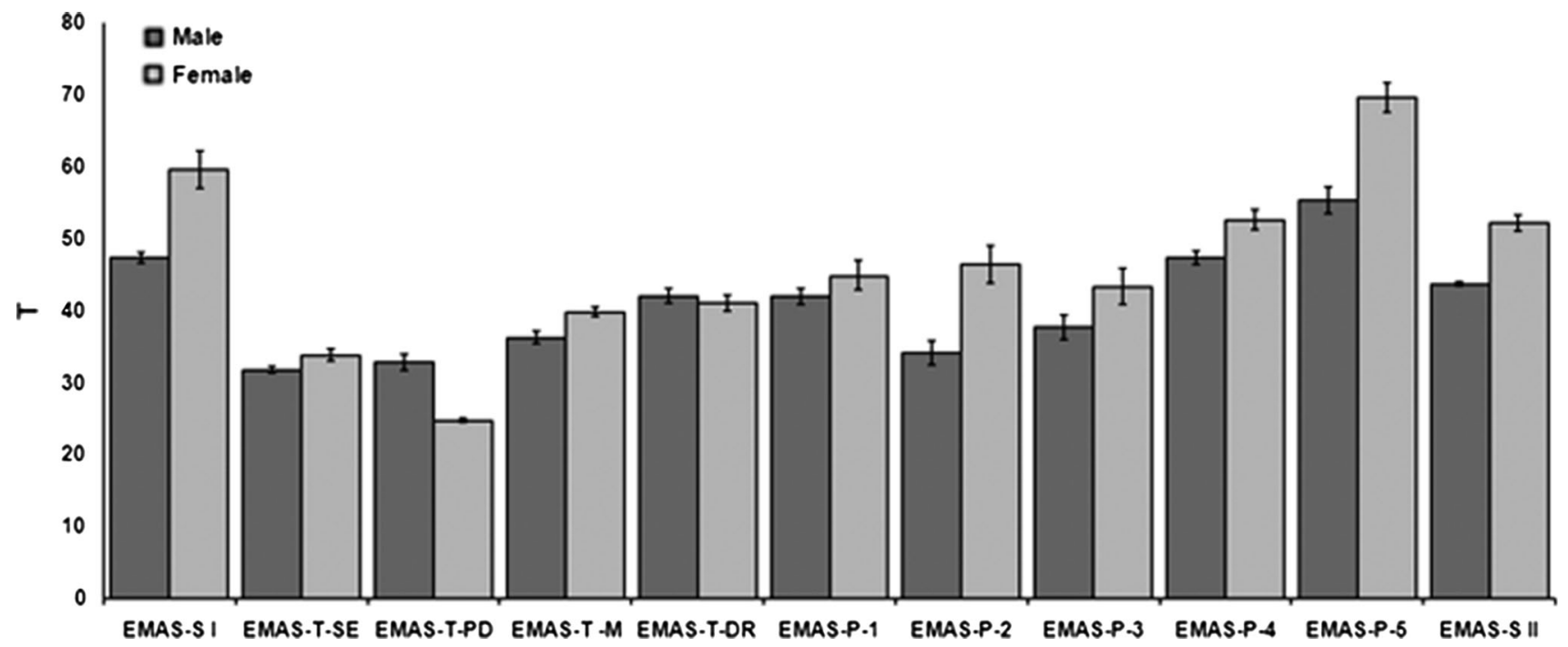

Fig. 2 EMAS test comparison by gender; bars represent confidence intervals at $99 \%$. EMAS-S I, state anxiety before CTCA; EMAS-T$\mathrm{SE}$, trait anxiety of social evaluation; EMAS-T-PD, trait anxiety of physical danger; EMAS-T-AM, trait anxiety of ambiguous situation; EMAS-T-DR, trait anxiety of daily routine; EMAS-P-1, perception of anxiety during SE; EMAS-P-2, perception of anxiety during PD; EMAS-P-3, perception of anxiety during AM; EMAS-P-4, perception of anxiety during DR; EMAS-P-5, perception of anxiety inherent in the present situation; EMAS-S II, state anxiety after CTCA consequently improves its applicability and spread. In this respect, the following missions are essential for the spread of a modern imaging technique: adequate information and preparation of the patient in a recovered doctor-patient relationship; the adoption of noninvasive investigations reducing associated anxiety and pain; less time spent in health facilities, with lower impact on the patients' psyche and work activities [28].

Several studies exist in the radiological literature that investigate the patients' acceptance of new noninvasive imaging methods [15-17]. It was demonstrated that noninvasive methods such as CT virtual colonoscopy [15], MR cholangiopancreatography [16], and MR angiography of the carotid arteries [17] are more widely accepted by patients than the traditional invasive approaches [18-23].

Computed tomography coronary angiography provides excellent results in the evaluation of atherosclerotic coronary artery disease due to high sensitivity and negative predictive value, which have enabled its clinical implementation [1-9]. The prognostic outcome [10-12] and the economic impact [29] of CTCA have been recently explored with extremely positive results. The effective 
strategies to reduce the dose of radiation introduced by the latest generation of scanners have helped to further improve the clinical applicability of CTCA [30].

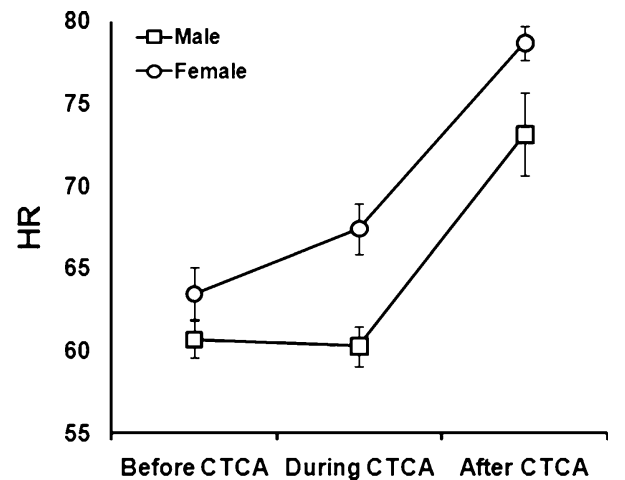

Fig. 3 Mean heart rate comparison by gender; bars represent confidence intervals at $99 \%$
In our study, the focus was instead oriented on examining the psychological impact of CTCA on the patient's anxiety and the acceptance of the technique. We used the EMAS test, a tool considered the gold standard for the assessment of anxiety in a clinical/hospital and research context, due to the excellent psychometric properties and the several validation studies of validity and reliability, as well as the presence of Italian standardised norms [31, 32]. The advantages of the EMAS test include: the ease and speed of administration, which is pivotal in a clinical/ hospital setting, where the patient is already unavoidably concerned about his own health and the medical procedure; a very accurate assessment of anxiety in relation to different contexts; the flexibility of using three measurement scales; the ability to apply the control of perceived anxiety of the situation (EMAS-P) to state anxiety scale (EMAS-S) [25].

Anxiety was more intense prior to CTCA than after, and higher in patients with a previous history of CCA. In

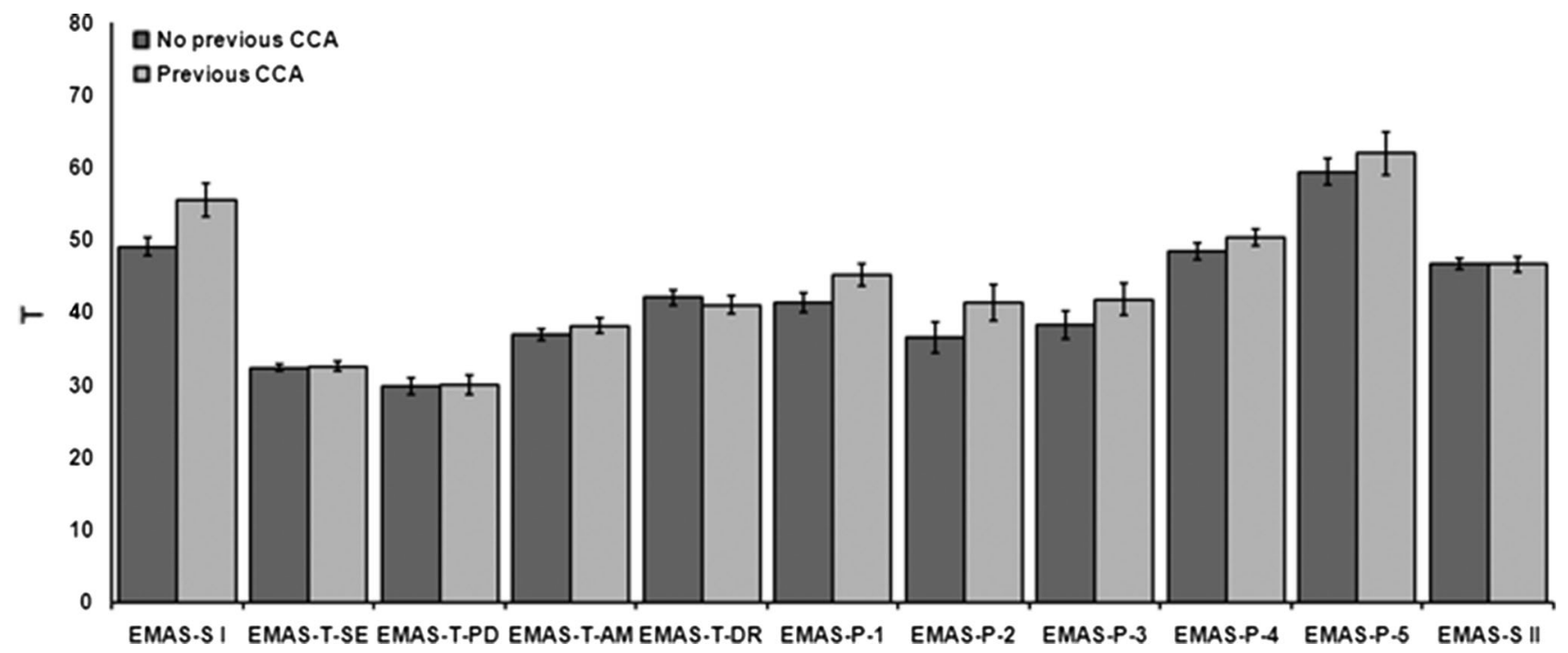

Fig. 4 EMAS test comparison of patients who underwent CTCA only versus patients with a history of CCA; bars represent confidence intervals at $99 \%$. EMAS-S I, state anxiety before CTCA; EMAS-TSE, trait anxiety of social evaluation; EMAS-T-PD, trait anxiety of physical danger; EMAS-T-AM, trait anxiety of ambiguous situation;
EMAS-T-DR, trait anxiety of daily routine; EMAS-P-1, perception of anxiety during SE; EMAS-P-2, perception of anxiety during PD; EMAS-P-3, perception of anxiety during AM; EMAS-P-4, perception of anxiety during DR; EMAS-P-5, perception of anxiety inherent in the present situation; EMAS-S II, state anxiety after CTCA

Table 1 Patients' acceptance of CTCA assessed with a five-point Likert scale questionnaire

\begin{tabular}{|c|c|c|c|c|c|}
\hline & 1. Very low & 2. Low & 3. Moderate & 4. High & 5. Very high \\
\hline Preparation & $0(0 \%)$ & $0(0 \%)$ & $9(2 \%)$ & $131(30 \%)$ & $302(68 \%)$ \\
\hline Concern & $60(14 \%)$ & $31(7 \%)$ & $29(6 \%)$ & $171(39 \%)$ & $151(34 \%)$ \\
\hline Comfort & $0(0 \%)$ & $0(0 \%)$ & $61(14 \%)$ & $200(45 \%)$ & $181(41 \%)$ \\
\hline Helplessness & $329(74 \%)$ & $40(9 \%)$ & $73(17 \%)$ & $0(0 \%)$ & $0(0 \%)$ \\
\hline Pain & $331(75 \%)$ & $29(7 \%)$ & $82(18 \%)$ & $0(0 \%)$ & $0(0 \%)$ \\
\hline Satisfaction & $0(0 \%)$ & $0(0 \%)$ & $0(0 \%)$ & $279(63 \%)$ & $163(37 \%)$ \\
\hline
\end{tabular}


Table 2 Patients' acceptance of CCA assessed with a five-point Likert scale questionnaire

\begin{tabular}{|c|c|c|c|c|c|}
\hline & 1. Very low & 2. Low & 3. Moderate & 4. High & 5. Very high \\
\hline Preparation & $20(11 \%)$ & $19(10 \%)$ & $10(6 \%)$ & $102(56 \%)$ & $30(17 \%)$ \\
\hline Concern & $0(0 \%)$ & $19(10 \%)$ & $21(12 \%)$ & $30(17 \%)$ & $111(61 \%)$ \\
\hline Comfort & $20(11 \%)$ & $21(11 \%)$ & $101(56 \%)$ & $39(22 \%)$ & $0(0 \%)$ \\
\hline Helplessness & $67(37 \%)$ & $33(18 \%)$ & $31(17 \%)$ & $50(28 \%)$ & $0(0 \%)$ \\
\hline Pain & $60(33 \%)$ & $41(23 \%)$ & $26(14 \%)$ & $19(11 \%)$ & $35(19 \%)$ \\
\hline Satisfaction & $18(10 \%)$ & $24(13 \%)$ & $52(29 \%)$ & $39(21 \%)$ & $48(27 \%)$ \\
\hline
\end{tabular}

women, anxiety and heart rate were higher than in men, affecting the image quality of CTCA, in agreement with the gender differences in diagnostic accuracy already reported in the literature [33]. CTCA had an excellent profile of acceptance by the patient, and better than CCA with respect to parameters such as preparation and information, comfort, degree of concern, helplessness, pain experienced, and overall satisfaction [14, 19].

There are a number of limitations to our study. The heart rate parameter was influenced by the pharmacological preparation (beta-blockers, benzodiazepines); however, diazepam was given before the administration of the EMAS test in order to equalise the heart rate-state anxiety readings. A proper and effective pharmacological preparation of patients represents an early essential step to perform CTCA [34, 35]. Image quality assessment was only performed on a per-patient basis to maintain patient unity in relation to individual psyche. Another limitation is that the surveys carried out with specific reference to CCA were exclusively performed in 181 patients on the basis of their previous medical history; the comparison was made only in this subgroup and not on a population undergoing both methods because of the compulsory containment of biological costs of ionising radiation [30].

Finally, it seems appropriate to emphasise that recovery of the traditional doctor-patient relationship in radiology should be built on the balance between diagnostic performance and patient acceptance, avoiding the consolidation of distorted alliances based on the use of defensive medicine, corporate interests, and patient self-referrals influenced by the mass-media [36-38].

\section{Conclusions}

From the point of view of a thorough and multidisciplinary evaluation of diagnostic imaging, CTCA, as seen by patients, proved to have an excellent acceptance profile. Higher levels of anxiety should be taken into account in women undergoing the examination, because they may partially affect image quality. The excellent acceptance profile of CTCA should be integrated with prognostic considerations and cost-effectiveness analyses in order to facilitate the clinical applicability of the technique.

Conflict of interest Ludovico La Grutta, Sabina La Grutta, Massimo Galia, Giuseppe Lo Piccolo, Giovanni Gentile, Giuseppe La Tona, Maria Stella Epifanio, Erica Maffei, Filippo Cademartiri, Rosa Lo Baido, Roberto Lagalla and Massimo Midiri declare no conflict of interest.

\section{References}

1. Raff GL, Gallagher MJ, O'Neill WW, Goldstein JA (2005) Diagnostic accuracy of noninvasive coronary angiography using 64-slice spiral computed tomography. J Am Coll Cardiol 46:552-557

2. Mollet NR, Cademartiri F, van Mieghem CA et al (2005) Highresolution spiral computed tomography coronary angiography in patients referred for diagnostic conventional coronary angiography. Circulation 112:2318-2323

3. Pugliese F, Mollet NR, Runza G et al (2006) Diagnostic accuracy of non-invasive 64-slice CT coronary angiography in patients with stable angina pectoris. Eur Radiol 16:575-582

4. Cademartiri F, Maffei E, Notarangelo F et al (2008) 64-slice computed tomography coronary angiography: diagnostic accuracy in the real world. Radiol Med 113:163-180

5. Malagutti P, Nieman K, Meijboom WB et al (2007) Use of 64-slice CT in symptomatic patients after coronary bypass surgery: evaluation of grafts and coronary arteries. Eur Heart $\mathrm{J}$ 28:1879-1885

6. Cademartiri F, Palumbo A, Maffei E et al (2007) Diagnostic accuracy of 64-slice CT in the assessment of coronary stents. Radiol Med 112:526-537

7. Cademartiri F, La Grutta L, Palumbo A et al (2007) Imaging techniques for the vulnerable coronary plaque. Radiol Med 112:637-659

8. Meijboom WB, Mollet NR, Van Mieghem CA et al (2006) Preoperative computed tomography coronary angiography to detect significant coronary artery disease in patients referred for cardiac valve surgery. J Am Coll Cardiol 48:1658-1665

9. Taylor AJ, Cerqueira M, Hodgson JM et al (2010) ACCF/SCCT/ ACR/AHA/ASE/ASNC/NASCI/SCAI/SCMR 2010 Appropriate Use Criteria for Cardiac Computed Tomography. A Report of the American College of Cardiology Foundation Appropriate Use Criteria Task Force, the Society of Cardiovascular Computed Tomography, the American College of Radiology, the American Heart Association, the American Society of Echocardiography, the American Society of Nuclear Cardiology, the North American Society for Cardiovascular Imaging, the Society for Cardiovascular Angiography and Interventions, and the Society for Cardiovascular Magnetic Resonance. Circulation 122:e525-e555 
10. Pundziute G, Schuijf JD, Jukema JW et al (2007) Prognostic value of multislice computed tomography coronary angiography in patients with known or suspected coronary artery disease. J Am Coll Cardiol 49:62-70

11. La Grutta L, Runza G, Gentile G et al (2011) Prognostic outcome of routine clinical noninvasive multidetector-row computed tomography coronary angiography in patients with suspected coronary artery disease: a 2-year follow-up study. Radiol Med 116:521-531

12. Maffei E, Seitun S, Palumbo A et al (2011) Prognostic value of Morise clinical score, calcium score and computed tomography coronary angiography in patients with suspected or known coronary artery disease. Radiol Med 116:1188-1202

13. Giovagnoni A, Bartolucci L, Manna A et al (2009) Health technology assessment: principles, methods and current status. Radiol Med 114:673-691

14. Schönenberger E, Schnapauff D, Teige F et al (2007) Patient acceptance of noninvasive and invasive coronary angiography. PLoS ONE 2:e246

15. Svensson MH, Svensson E, Lasson A, Hellstrom M (2002) Patient acceptance of CT colonography and conventional colonoscopy: prospective comparative study in patients with or suspected of having colorectal disease. Radiology 222:337-345

16. Menon K, Barkun AN, Romagnuolo J et al (2001) Patient satisfaction after MRCP and ERCP. Am J Gastroenterol 96:2646-2650

17. U-King-Im JM, Trivedi R, Cross J et al (2004) Conventional digital subtraction $\mathrm{x}$-ray angiography versus magnetic resonance angiography in the evaluation of carotid disease: patient satisfaction and preferences. Clin Radiol 59:358-363

18. Mikosh P, Hadrawa T, Laubreiter K et al (2010) Effectiveness of respiratory sinus arrhythmia biofeedback on state anxiety in patient undergoing coronary angiography. J Adv Nurs 66:1101-1110

19. Heikkila J, Paunonen M, Laippala P, Virtanen V (1999) Patient's fears in coronary arteriography. Scand J Caring Sci 13:3-10

20. Uzun S, Vural H, Uzun M, Yokosoglu M (2008) State and trait anxiety levels before coronary angiography. J Clin Nurs 17:602-607

21. De Jong-Watt WJ, Arthur HM (2004) Anxiety and health related quality of life in patients awaiting elective coronary angiography. Hearth Lung 33:237-248

22. Closa Leon T, Nouwen A, Sheffield D et al (2010) Anger rumination, social support and cardiac symptoms in patients undergoing angiography. Br J Health Psychol 15:841-857

23. Weeks BP, Nilsson U (2011) Music interventions in patients during coronary angiographic procedures: a randomized controlled study of the affect on patients anxiety and well being. Eur J Cardiovasc Nurs 10:88-93
24. Runza G, La Grutta L, Alaimo V et al (2008) Influence of heart rate in the selection of the optimal reconstruction window in routine clinical multislice coronary angiography. Radiol Med 113:644-657

25. Endler NS, Parker JD, Bagby RM, Cox BJ (1991) Multidimensionality of state and trait anxiety: factor structure of the Endler Multidimensional Anxiety Scales. J Pers Soc Psychol 60:919-926

26. Likert R (1932) A technique for the measurement of attitudes. Arch Psychol 140:44-53

27. Hunink MGM, Krestin GP (2002) Study design for concurrent development, assessment and implementation of new diagnostic imaging technology. Radiology 222:604-614

28. Battista RN, Hodge MJ, Vineis P (1995) Medicine, practice and guidelines: the uneasy juncture of science and art. J Clin Epidemiol 7:875-880

29. Stacul F, Sironi D, Grisi G et al (2009) 64-Slice CT coronary angiography versus conventional coronary angiography: activitybased cost analysis. Radiol Med 114:239-252

30. Maffei E, Martini C, De Crescenzo S et al (2010) Low dose CT of the heart: a quantum leap into a new era of cardiovascular imaging. Radiol Med 115:1179-1207

31. Gustavsson-Lilius M, Julkunen J, Keskivaara P et al (2012) Predictors of distress in cancer patients and their partners: the role of optimism in the sense of coherence construct. Psychol Health 27:178-195

32. McQuillan J, Fifield J, Sheehan TJ et al (2003) A comparison of self-reports of distress and affective disorder diagnoses in rheumatoid arthritis: a receiver operator characteristic analysis. Arthr Rheum 49:368-376

33. Meijboom WB, Weustink AC, Pugliese F et al (2007) Comparison of diagnostic accuracy of 64-slice computed tomography coronary angiography in women versus men with angina pectoris. Am J Cardiol 100:1532-1537

34. Maffei E, Palumbo AA, Martini C et al (2009) "In-house" pharmacological management for computed tomography coronary angiography: heart rate reduction, timing and safety of different drugs used during patient preparation. Eur Radiol 19:2931-2940

35. Cubuk R, Tasali N, Yilmazer S et al (2011) Effect of an oral anxiolytic medication and heart rate variability on image quality of 64-slice MDTC coronary angiography. Radiol Med 116:47-55

36. Mitchell JM (2008) Utilization trends for advanced imaging procedures: evidence from individuals with private insurance coverage in California. Med Care 46:460-466

37. Suter LG, Elmore JG (1998) Self-referral for screening mammography. J Gen Intern Med 13:710-713

38. Illes J, Kann D, Karetsky K et al (2004) Advertising, patient decision making, and self-referral for computed tomographic and magnetic resonance imaging. Arch Intern Med 164:2415-2419 